\title{
Doppler images of DI Piscium during 2004-2006*
}

\author{
M. Lindborg ${ }^{1}$, T. Hackman ${ }^{1,2}$, M. J. Mantere ${ }^{3}$, H. Korhonen ${ }^{2,4}$, I. Ilyin $^{5}$, O. Kochukhov ${ }^{6}$, and N. Piskunov ${ }^{6}$ \\ 1 Department of Physics, PO Box 64, 00014 University of Helsinki, Finland \\ e-mail: marjaana.lindborg@helsinki.fi \\ 2 Finnish Centre for Astronomy with ESO (FINCA), University of Turku, Väisäläntie 20, 21500 Piikkiö, Finland \\ 3 Aalto University, Department of Information and Computer Science, PO Box 15400, 00076 Aalto, Finland \\ ${ }^{4}$ Niels Bohr Institute, Juliane Maries Vej 30, 2100 University of Copenhagen, Denmark \\ 5 Leibniz Institute for Astrophysics (AIP), an der Sternwarte 16, 14882 Potsdam, Germany \\ ${ }^{6}$ Department of Physics and Astronomy, Uppsala University, Box 516, Uppsala 751 20, Sweden
}

Received 14 September 2013 / Accepted 15 January 2014

\begin{abstract}
Aims. DI Psc (HD 217352) is a Li-rich, rapidly rotating single K giant. We set out to study the spot configuration and activity level by calculating surface temperature maps of the star.

Methods. We apply the Doppler imaging method on high-resolution optical spectroscopy obtained during 2004-2006.

Results. In July-August 2004, no clear spot structures were visible, but the spot coverage increased in July 2005, and cool spots emerged, especially at intermediate latitudes. Later on in September 2006, the spot coverage increased and cool spots were visible on both sides of the equator. However, the map of 2006 suffers from bad phase coverage, meaning it is not possible to draw definite conclusions on the spot locations during that season.

Conclusions. Compared with earlier Doppler maps of DI Psc and temperature maps obtained for other late-type stars with similar rotation rates, DI Psc seems to be in a low activity state especially during the observing season of July-August 2004. During the 2005 and 2006 observing seasons, the spot activity seen in the spectral line profiles and inferred from Doppler images increases, and the temperature contrast in our last map is more comparable to what was reported in an earlier study. Therefore, it can be concluded that the spot activity level of the star is variable over time. However, the present and previous Doppler images form too short a time series to draw conclusions about a possible activity cycle in DI Psc.
\end{abstract}

Key words. stars: individual: HD 217352 - stars: activity - stars: imaging - starspots

\section{Introduction}

DI Piscium (HD 217352) is one of the low-mass lithium-rich [LTE $\log n=2.20] \mathrm{K} 1$ giants (Kővári et al. 2013) with a rotation period of approximately 18 days (Strassmeier et al. 2000; Koen \& Eyer 2002; Kővári et al. 2013). It has been stated that the rare population of Li-rich $\mathrm{K}$ giants is composed of objects rotating faster than ordinary giants of the same type (e.g. Reddy \& Lambert 2005; Drake et al. 2002). DI Psc is one of the fastest rotators within this population, and more studies are still needed to determine whether the angular momentum is added to the envelope from the interior (rapidly rotating core) or from outside (merging planet), or if it is an effect of merged binaries (Drake et al. 2002).

The cause for the anomalously high Li abundances in this class of objects also remains under debate. Lithium is destroyed within a star's main sequence phase and can mainly survive in a thin layer at the surface of the star due to the lack of mixing between this and other layers. Observations of $\mathrm{Li}$ abundances in $\mathrm{K}$ giants confirm that the values observed are close to the predicted upper limit (e.g. Melo et al. 2005; Kumar et al. 2011), and in some objects even exceed the present interstellar medium

* Based on observations made with the Nordic Optical Telescope, operated on the island of La Palma jointly by Denmark, Finland, Iceland, Norway, and Sweden in the Spanish Observatorio del Roque de los Muchachos of the Instituto de Astrofísica de Canarias. value (see e.g. de La Reza \& da Silva 1995; Balachandran et al. 2000).

Charbonnel \& Balachandran (2000) describe two methods of lithium production. The low-mass red giant branch (RGB) stars, which go through a helium flash, produce Li at the phase seen as a bump in the luminosity function. When the outwardmoving hydrogen-burning shell first meets the convective envelope owing to the increasing helium core mass, the surface luminosity is decreased, because the availability of extra hydrogen decreases the mean molecular weight. The outwardly moving hydrogen shell burns through the mean molecular weight discontinuity (Charbonnel \& Balachandran 2000), and extra mixing processes, such as the one induced by rotation, can now connect helium-rich envelope material to the outer regions of the hydrogen-burning shell, which activates Li production. The vast majority of Li-rich $\mathrm{K}$ giants have luminosity and effective temperature combinations that permit Li production at a helium core flash, which has been proposed as another possible mechanism for producing the Li-rich giant population (see e.g. Kumar et al. 2011). The main cause of these abnormal abundances of $\mathrm{Li}$ in low-mass red giant stars still remains unknown (e.g. Kumar et al. 2011), since none of the above-mentioned scenarios are directly supported by observational evidence. For instance, even though the Li-rich RGB stars rotate faster than average, high Li abundances have been observed in slow rotators, and low abundances in fast rotators, casting some doubt on the importance of the extra mixing provided by rotation. 
Recently, DI Psc has also been studied using the Doppler imaging technique by Kővári et al. (2013). These results suggest that the star shows large cool spots both in polar and low-latitude regions. Such cool spots are generally interpreted as manifestations of the presence of strong magnetic fields, hence a sign of magnetic activity driven by an underlying dynamo mechanism. The operation of a dynamo relies on the collective inductive action of rapid enough rotation, convective turbulence, and non-uniformities in the velocity field (see e.g. Brandenburg \& Subramanian 2005).

Observational studies of other rapidly rotating late-type stars with convective envelopes have shown that these stars, fairly independently of spectral type or binarity, consistently show similar types of spot configurations on their surfaces (see the reviews by Strassmeier 2009; Berdyugina 2005). The spots are usually large, and they occur at high latitudes, sometimes even in the polar regions. Moreover, the spot distribution over longitude is uneven, which has led to the conclusion that the dynamo mode in question is non-axisymmetric. In some of the observed giant stars, e.g. in the RS CVn binary system primary component II Peg, the spots are observed to drift with respect to the surface rotation of the star (Lindborg et al. 2011; Hackman et al. 2011, 2012; Lindborg et al. 2013). This can be understood as an azimuthal dynamo wave of the non-axisymmetric field (see e.g. Mantere et al. 2013, and references therein). Similar behaviour, but with a less systematic drift, has been reported in the single giant FK Com (Korhonen et al. 2007; Hackman et al. 2013). The magnetic activity in these objects, therefore, manifests itself very differently than in the Sun, where the magnetic field is mostly axisymmetric (see e.g. Pelt et al. 2006, and references therein), and the toroidal field shows latitudinal migration causing the well-known butterfly diagram of sunspots (see e.g. Ossendrijver 2003, and references therein).

Since the Doppler imaging study of Kővári et al. (2013) presents only one observing season of the object, it is desirable to extend their study in time to investigate the evolution of the cool spots on the star's surface. DI Psc was spectroscopically monitored with the SOFIN spectrograph at the Nordic Optical Telescope (La Palma, Spain) during the years 2004-2006. In this paper we compute three new surface temperature maps for DI Psc based on SOFIN at NOT observations. In Sect. 2 we describe our dataset, in Sect. 3 the Doppler imaging method. In Sect. 4 we present the new Doppler images derived, and finally in Sect. 5 discuss the implications of our results.

\section{Spectroscopic observations with SOFIN}

All the observations were made using the SOFIN highresolution échelle spectrograph at the $2.56 \mathrm{~m}$ Nordic Optical Telescope (NOT), La Palma, Spain. The data was acquired with the second camera equipped with a Loral CCD detector containing $2048 \times 2048$ pixels. This gives a spectral resolution of $R \approx 70000$.

A total of three sets of high-resolution spectra of DI Psc were measured in 2004-2006 (July-August 2004, July 2005, and September 2006), with signal-to-noise ratios (S/N) usually around 200-300, so in this paper we publish three new Doppler imaging temperature maps. The amount of observed phases varied from 5 (September 2006) to 12 (July-August 2004). The spectral region of 6410-6440 Å was chosen for Doppler imaging because of the presence of strong enough and well-known lines. That we include the ionized iron line FeII $6432.68 \AA$ helps to constrain the average temperature of the star by using the ionization balance of neutral and ionized Fe lines. The observations
Table 1. Observations with NOT.

\begin{tabular}{lccc}
\hline \hline $\begin{array}{l}\text { Date } \\
\text { (dd/mm/yyyy) }\end{array}$ & HJD & $\phi$ & $S / N$ \\
\hline $29 / 07 / 2004$ & 3215.657 & 0.27 & 212 \\
$31 / 07 / 2004$ & 3217.701 & 0.39 & 228 \\
$01 / 08 / 2004$ & 3218.604 & 0.44 & 290 \\
$02 / 08 / 2004$ & 3219.759 & 0.50 & 149 \\
$03 / 08 / 2004$ & 3220.617 & 0.55 & 207 \\
$05 / 08 / 2004$ & 3222.663 & 0.66 & 269 \\
$06 / 08 / 2004$ & 3223.697 & 0.72 & 264 \\
$07 / 08 / 2004$ & 3224.714 & 0.77 & 195 \\
$08 / 08 / 2004$ & 3225.665 & 0.83 & 208 \\
$09 / 08 / 2004$ & 3226.702 & 0.88 & 263 \\
$10 / 08 / 2004$ & 3227.673 & 0.94 & 253 \\
$11 / 08 / 2004$ & 3228.699 & 0.99 & 204 \\
\hline $15 / 07 / 2005$ & 3566.641 & 0.70 & 261 \\
$17 / 07 / 2005$ & 3568.622 & 0.81 & 343 \\
$19 / 07 / 2005$ & 3570.585 & 0.92 & 264 \\
$22 / 07 / 2005$ & 3573.708 & 0.09 & 236 \\
$23 / 07 / 2005$ & 3574.567 & 0.14 & 266 \\
$29 / 07 / 2005$ & 3580.712 & 0.48 & 255 \\
\hline $02 / 09 / 2006$ & 3980.640 & 0.62 & 220 \\
$06 / 09 / 2006$ & 3984.680 & 0.84 & 225 \\
$09 / 09 / 2006$ & 3987.664 & 0.00 & 242 \\
$11 / 09 / 2006$ & 3989.626 & 0.11 & 220 \\
$13 / 09 / 2006$ & 3991.613 & 0.22 & 200 \\
\hline
\end{tabular}

Notes. The heliocentric Julian date is given as HJD-2 450 000, and rotational phases are calculated using Eq. (4).

are summarized in Table 1, where we give the details of the individual observations.

Observations of approximately ten evenly distributed rotation phases are regarded as optimal for Doppler imaging (Vogt et al. 1987). Thus the observation from 2006 definitely has insufficient phase coverage. Vogt et al. (1987) examined the effect of incomplete phase coverage on the images: the effect of poor sampling was found similar to low $\mathrm{S} / \mathrm{N}$, the main problem being errors and fuzziness in the spot shapes. The spot locations and sizes were quite well recovered. Rice \& Strassmeier (2000) concluded that a phase gap as large as $100^{\circ}$ does not significantly affect the recovered features. Doppler images based on observation with larger phase gaps than this have been published before, e.g. in the studies of II Peg (Berdyugina et al. 1998), EI Eri (Washuettl et al. 2009), and HD 171488 (Jeffers et al. 2011). The largest phase gap in the observations from 2006 corresponds to roughly $160^{\circ}$. We can thus expect that the insufficient phase coverage in the observations will result in errors in, say, the shape and latitudes of the spots, but we will still get useful information about the spot coverage.

As for the $\mathrm{S} / \mathrm{N}$, we stress that we use a synthesis of several lines in our analysis. This means that noise is partly cancelled out, and the resulting temperature maps are not as sensitive to noise as in the tests performed by e.g. Vogt et al. (1987).

The spectral observations were reduced with the 4A software system (Ilyin 2000). Bias, cosmic ray, flat field and scattered light corrections, wavelength calibration and normalization, and corrections for the motion of the Earth were included in the reduction process. 


\section{Doppler imaging}

We use the Doppler imaging code INVERS7 written by Piskunov (Piskunov et al. 1990; Piskunov 1991). In INVERS7, the ill-posed inversion problem is solved by Tikhonov regularization. Generally this means introducing an additional structural constraint to minimize the surface gradient of the solution. The Doppler imaging solution is retrieved using a table of calculated local line profiles for different surface positions (limb angles) and different effective temperatures.

Local line profiles are calculated using numerical models of stellar atmospheres. The choice of parameters used is not trivial, because different parameters have similar effects on the spectral lines, and appropriate fits can be obtained by using different combinations of stellar and spectral parameters. The inversion procedure is very sensitive to changes in the parameter describing microturbulence, so that varying it causes shifts in the average effective temperature $T_{\text {eff }}$ of the solution. Making errors in the calculations and using an inaccurate $v \sin i$ can produce artefacts, seen as cool polar caps, and hot or cool belts in the Doppler imaging maps. Thus, establishing the connection between the variation in the stellar parameters and the line profiles is crucial (e.g. Lindborg et al. 2011; Hackman et al. 2012).

The inversion method is described in more detail in the papers by Piskunov et al. (1990) and Piskunov (1991) and in our previous study of II Peg (Lindborg et al. 2011).

\subsection{Stellar parameters}

Setting the stellar parameters for single stars is more demanding than for close binaries. In the latter case the inclination can be deduced from the orientation of the binary orbits, while in the former, the inclination is often roughly estimated from known rotation periods, the $v \sin i$, and an assumed radius. Vogt et al. (1987) made tests of how different (wrongly chosen) inclinations influence the Doppler imaging result. Their conclusion was that the overall locations of spots and shapes of spots are well preserved and insensitive to errors.

DI Psc is located at a distance of $190_{-23}^{+30}$ parsecs from the solar system (van Leeuwen 2007). The spectral type of DI Psc has been determined to be K1 III giant. According to Gray (2008) this gives the effective temperature $T_{\text {eff }} \approx 4700 \mathrm{~K}$ and the surface gravity $\log g \approx 3$. In the variable star one-shot project VSOP (Dall et al. 2007) DI Psc was reclassified as a G8III-IV spectral type, and claimed to be a double-lined spectroscopic binary. We examined the spectra, but could not find any traces of orbital motion in the spectra. Kővári et al. (2013) calculated heliocentric velocities for DI Psc from observations and determined the velocity to be around $-18 \mathrm{~km} \mathrm{~s}^{-1}$ in all sets of observations. Based on this we conclude that DI Psc is a single star, not a spectroscopic binary.

In the analysis presented here, we used the MARCS planeparallel LTE stellar model atmospheres (Gustafsson et al. 2008) with temperatures $3000-6000 \mathrm{~K}$. We adopted a radial-tangential macroturbulence of $\zeta_{\mathrm{t}}=4.0 \mathrm{~km} \mathrm{~s}^{-1}$ from Gray (2008).

The microturbulence $\xi_{\mathrm{t}}$ and inclination $i$ were determined by testing different values. The best fit was achieved with $i=60^{\circ}$ and $\xi_{\mathrm{t}}=2.0 \mathrm{~km} \mathrm{~s}^{-1}$. All the stellar parameters used in the inversions are summarized in Table 2.

According to Strassmeier et al. (2000), the rotation period would be $P \approx 18.4$, and according to HIPPARCos photometry, $P \approx 18^{\mathrm{d}}$ (Koen \& Eyer 2002). Kővári et al. (2013) analyzed rotational properties from photometry, which led to the more accurate period of $P \approx 18.066 \pm 0.088$. We re-estimated the period
Table 2. Adopted values of the stellar parameters of DI Psc.

\begin{tabular}{lc}
\hline \hline Parameter & Adopted value \\
\hline$\xi_{\mathrm{t}}$ & $2.0 \mathrm{~km} \mathrm{~s}^{-1}$ \\
$\zeta_{\mathrm{t}}$ & $4.0 \mathrm{~km} \mathrm{~s}^{-1}$ \\
$\log g$ & 3.0 \\
$P_{\text {orb }}$ & $18.066 \mathrm{~d}$ \\
$v \sin i$ & $41.0 \mathrm{~km} \mathrm{~s}^{-1}$ \\
$i$ & $60^{\circ}$ \\
$T_{\text {eff }}$ & $4700 \mathrm{~K}$ \\
$\log \left[n_{\mathrm{Fe}} / n_{\text {tot }}\right]$ & -4.55 \\
$\left.\log \left[n_{\mathrm{Ca}} / n_{\text {tot }}\right]\right]$ & -5.68 \\
$\log [\mathrm{M} / \mathrm{H}]$ & 0.0 \\
\hline
\end{tabular}

with the re-formulated Barnes-Evans relation (Beuermann et al. 1999). Observational values of absolute $V$-band magnitude $M_{V}=0.47$ and de-reddened $V-I_{\mathrm{C}}=1.05$ were adopted from Kővári et al. (2013). The Barnes-Evans relation by Beuermann et al. (1999) yields

$S_{V}=M_{V}+5 \log \left(R / R_{\odot}\right)$,

where $S_{V}$ is a surface brightness of the star in $V$-band, which can be estimated for giant stars

$S_{V}=4.71( \pm 0.14)+1.74( \pm 0.05)\left(V-I_{\mathrm{C}}\right)$.

From these relations we can compute the radius of the star, yielding roughly $R \approx 16.3 R_{\odot}$. Thus, the rotation period of the star can be obtained in the following way:

$P_{\text {rot }}=\frac{2 \pi R \sin i}{v \sin i} \approx 17.5 \mathrm{~d}$

where we have used the optimal value of inclination, $i=60^{\circ}$, in our analysis. In conclusion, the rotation period of Kővári et al. (2013) of $P=18$ d.066 is in acceptable agreement with our estimations. Rotational phases were calculated using the ephemerid:

$\mathrm{HJD}=2451639.00330+18.066 \times E$,

where the epoch is the time of the first used spectral observation in Kövári et al. (2013), and the period is the photometric period.

\subsection{Spectral parameters}

The following spectral lines were used in the surface temperature inversions: Fe I $6411.649 \AA$ A, Fe I $6430.846 \AA$, Fe II $6432.680 \AA$, and Ca I $6439.075 \AA$. All these lines are actually blends of several lines. In total 43 lines were included in the calculations. The spectral line parameters were obtained from the Vienna Atomic Line Database (Kupka et al. 1999). Solar element abundances were used $(\log [\mathrm{M} / \mathrm{H}]=0.0)$. The $\log (g f)$ values of two Fe I lines, one Fe II line, and one Ca I line, i.e. the strongest lines, were modified from the standard values, shown in Table 3, because the standard values were found to produce a wrong balance between these lines. We note that also Kővári et al. (2013) encountered problems in fitting some photospheric lines for DI Psc.

\section{Results}

Our observations were made during the years 2004-2006, with one observing run during each year. This allows us to calculate three new Doppler imaging temperature maps for the star. 
Table 3. Abundance adjustments for standard spectral parameters.

\begin{tabular}{lrr}
\hline \hline Line & $\log (g f)_{\text {standard }}$ & $\log (g f)_{\text {adopted }}$ \\
\hline Fe I 6411.649 & -0.745 & -0.945 \\
Fe I 6430.846 & -2.129 & -1.910 \\
Fe II 6432.680 & -3.640 & -3.840 \\
Ca I 6439.075 & 0.100 & 0.150 \\
\hline
\end{tabular}

Table 4. Average temperature $T_{\text {mean }}$, temperature difference $\Delta T=$ $T_{\text {mean }}-T_{\min }$, spot filling factor $F_{\mathrm{S}}$, and deviation $d$ of the Doppler imaging solution in the obtained inversion maps.

\begin{tabular}{lccrc}
\hline \hline Season & $T_{\text {mean }}[\mathrm{K}]$ & $\Delta T[\mathrm{~K}]$ & $F_{\mathrm{S}}[\%]$ & $d\left[10^{-5}\right]$ \\
\hline Jul.-Aug. 04 & 4690 & 270 & 0.0 & 6.19 \\
Jul. 05 & 4700 & 553 & 21.3 & 5.52 \\
Sep. 06 & 4690 & 590 & 35.2 & 5.56 \\
\hline
\end{tabular}

Table 4 lists the average temperature computed over the visible surface, the temperature difference between the coolest surface structures and the average temperature, and the spot filling factor when the threshold for cool spots is taken as $T=4400 \mathrm{~K}$.

In July-August 2004 (see Fig. 1), no clearly detectable spot regions are visible, and the temperature differences between the cool and warmer areas are around $300 \mathrm{~K}$. The spot filling factor is zero, so DI Psc is effectively spotless at this time. About one year later in July 2005 (the second temperature map shown in Fig. 1), cool spots have appeared at both high and low latitudes. The coolest spot structure extends from latitudes $30^{\circ}-60^{\circ}$. The spot activity has increased dramatically since the spot filling factor is $21.3 \%$. In September 2006 (Fig. 2), the spots can be seen at a wider latitude range, with weak spots both at the polar regions and below the equator. The spot filling factor has increased further to $35.2 \%$.

The spot structures are weak in 2004, the temperature contrast between the cool spots and the average temperature being less than $300 \mathrm{~K}$, but increasing over time. During July 2005, the spots are roughly $553 \mathrm{~K}$ cooler, and $590 \mathrm{~K}$ cooler during the 2006 season than the average.

Our September 2006 image has rather low phase coverage, and some spots are centred on phases where we do not have any observations, so it is hard to say whether those spots are real or artefacts. However, we want to stress that the presence of spots is clearly seen from bumps in spectral lines in the observations from 2006. Furthermore, as demonstrated by e.g. Vogt et al. (1987) and Rice \& Strassmeier (2000), poor phase coverage mainly affects the positions and shapes of spots, but should not introduce spurious spot structures. We thus regard the spot filling factors as, if not completely accurate, at least directional; nevertheless, in the next section we test the reliability of the poorest phase coverage map in detail.

\subsection{Reliability of the September 2006 map}

To estimate the errors due to poor phase coverage we tested how insufficient phase coverage shapes the July-August 2004 map by removing several phases so that only five phases, corresponding roughly to our September 2006 map, were left in the inversion. The result is displayed in Fig. 3. The most prominent artefact is the cool and hot spot structure centred on phase 0.9. In the original image there was only a very weak cool spot at this position. The test shows that the spot filling factor increases slightly when the phase coverage is reduced. In the test the spot coverage factor rises to $4.8 \%$, and temperature difference is $400 \mathrm{~K}$. Thus we state that with only five observations we still can have a realistic estimate of the spot coverage.

\section{Summary and discussion}

We publish three new temperature maps for DI Psc during 2004-2006. Our map of July-August 2004, which is our best observing season with high phase coverage, reveals a more or less spotless appearance. This is a dramatic change from the May 2000 surface temperature maps published by Kóvári et al. (2013), showing large polar and belt-like low-latitude spot with a significant temperature contrast. Although the inversion method and some adopted stellar parameters (such as the inclination) were different from ours, the observations were also of good quality with sufficient phase coverage. Therefore, both of the maps can be regarded as almost equally reliable, and the differences are therefore unlikely to be caused by the aforementioned facts.

Although with poorer phase coverage, our maps for the 2005 and 2006 seasons seem to indicate recovering spot activity, with both high-latitude and low-latitude spots appearing on the stellar surface. Although the spot distribution resembles that of the May 2000 Doppler image in Kővári et al. (2013), the temperature contrasts for the September 2006 observing season map is still lower than the $\Delta T \sim 1000 \mathrm{~K}$ reported by Kővári et al. (2013). This map was derived with another Doppler imaging code and different stellar and spectral parameters, but these differences should show up more as axisymmetric structures than individual spurious spots. Furthermore, the cooler spots retrieved by Konvári et al. (2013) are supported by the stronger bumps seen in their spectral lines.

The combined view arising from all the so-far produced surface temperature maps of DI Psc (Kővári et al. 2013, and this study) suggests that the spot coverage fraction has varied strongly on a time scale of a few years. The strong spot activity observed in May 2000 practically vanished within the four years to July-August 2004. After this the spot activity regained some of its strength until September 2006. This evolution is not only supported by our Doppler images, but can be traced directly from distortions in the spectral lines.

The spot evolution can be a consequence of an activity cycle, but the follow-up time is too short for any conclusion on any periodicities in the activity level. Of course, the Doppler images are just snapshots in time, so faster fluctuations in activity are not ruled out.

Lithium-rich, rapidly rotating single giants are a hot topic both in observational and theoretical astrophysics. The cause of high Li-abundances in $\mathrm{K}$ giants still remains under debate. The stellar parameters for DI Psc are still not well known, which is a severe drawback for constructing reliable surface temperature maps using Doppler imaging techniques. Even the spectral type and single star status of DI Psc have been questioned. We checked several observing sets and different wavelength regions, but no marks of a spectroscopic binary component was detected, so we claim that DI Psc is a K-type single giant.

The convective turnover time, $\tau_{\mathrm{c}}$, of $\mathrm{G}-\mathrm{K}$ giants in the luminosity classes III-IV can be estimated to be on average $50 \mathrm{~d}$ (see e.g. Saar \& Brandenburg 1999, Table 4, and references therein). The inverse Rossby number, also called the Coriolis number, which characterizes the strength of the rotational influence on the turbulent motions in the stellar convection zone, makes it possible to theoretically predict activity cycle lengths in rapid rotators. Using this rough estimate of $\tau_{c}$, would give 
M. Lindborg et al.: Doppler images of DI Piscium during 2004-2006
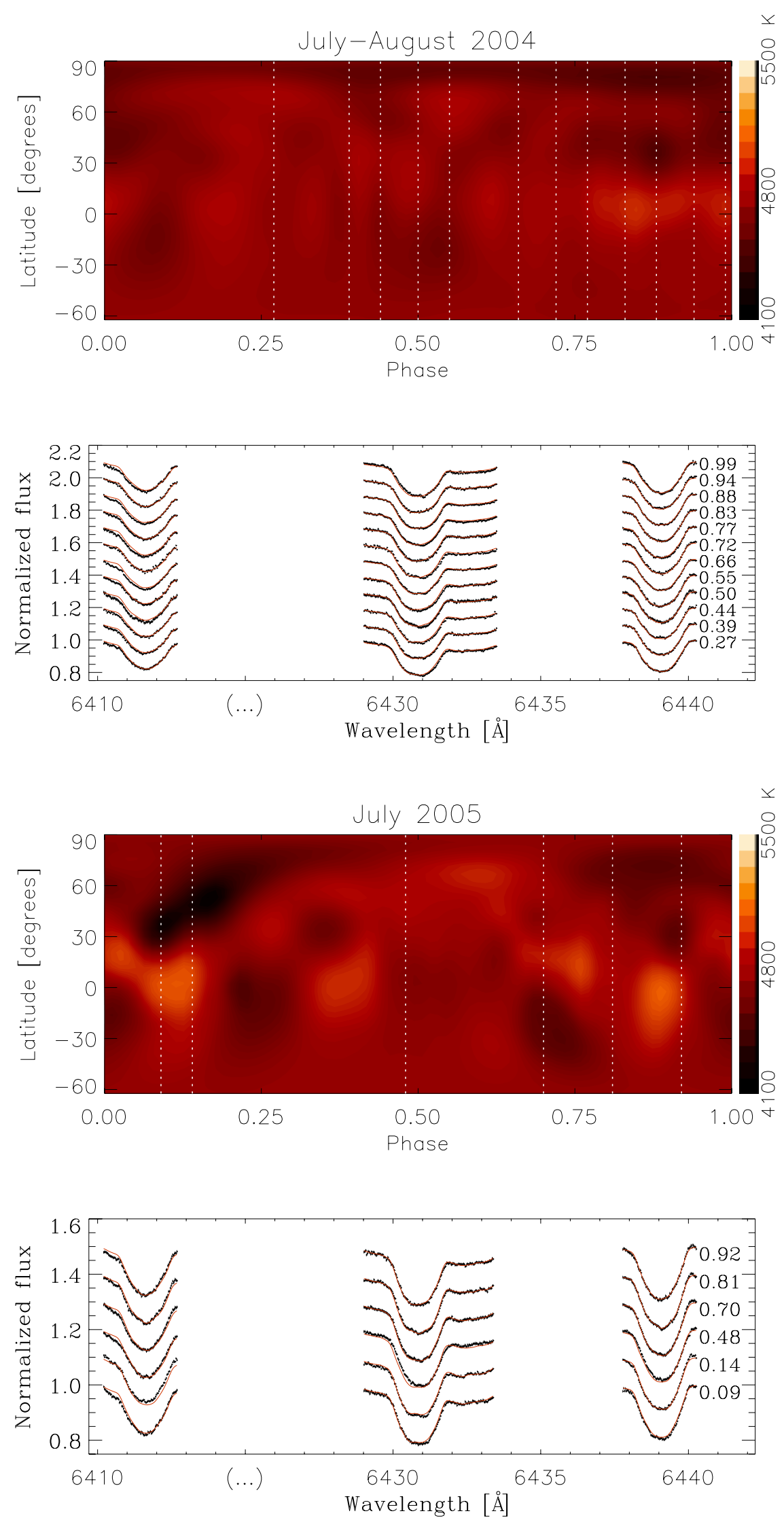

Fig. 1. Doppler images in July-August 2004 and July 2005. Upper panel of each pair: equirectangular projection of the surface temperature distribution obtained. Lower panel: observed (vertical lines, the height representing the errors in observations) and calculated line profiles (solid lines). 
A\&A 562, A139 (2014)
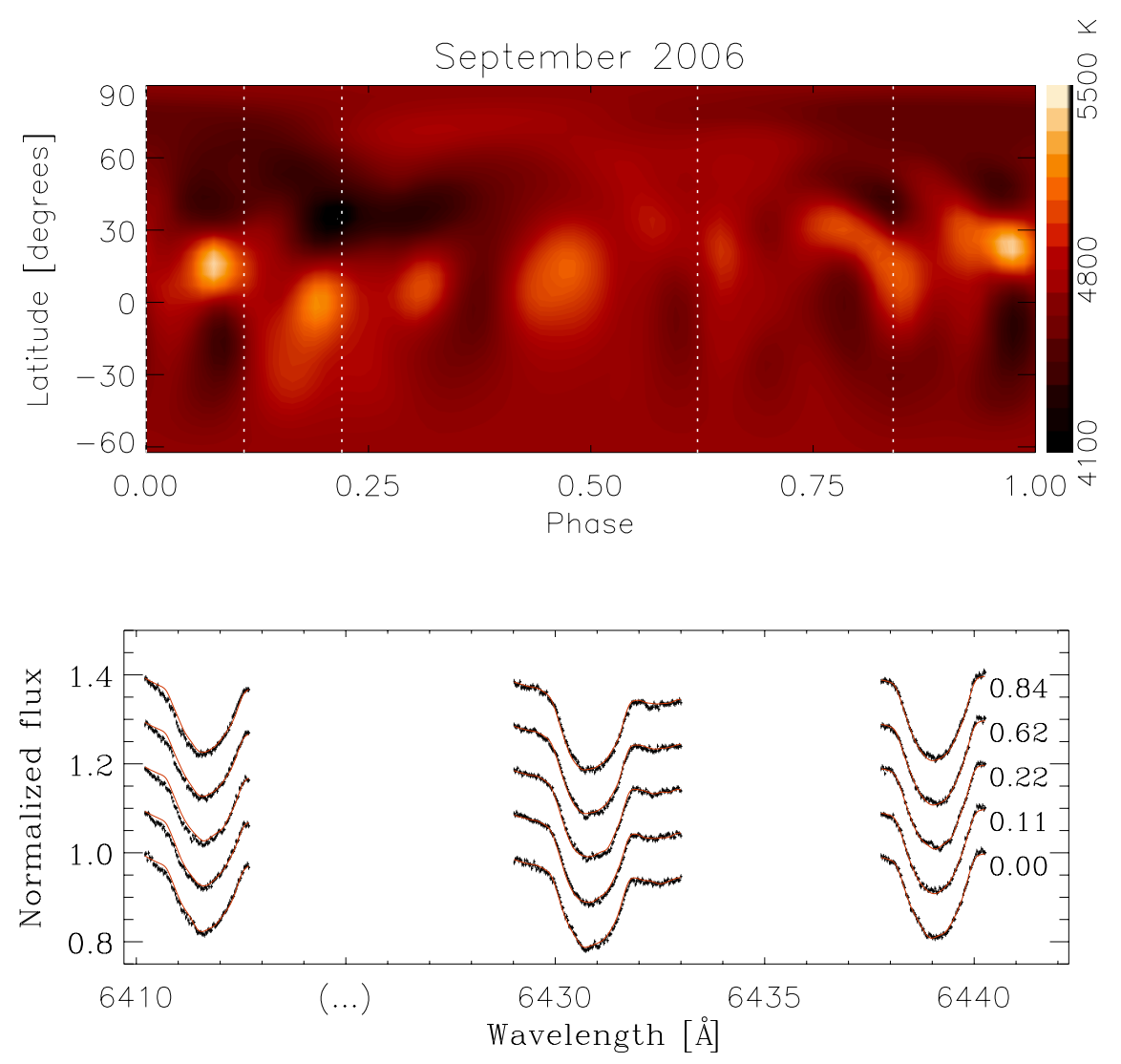

Fig. 2. Same as in Fig. 1 for September 2006.
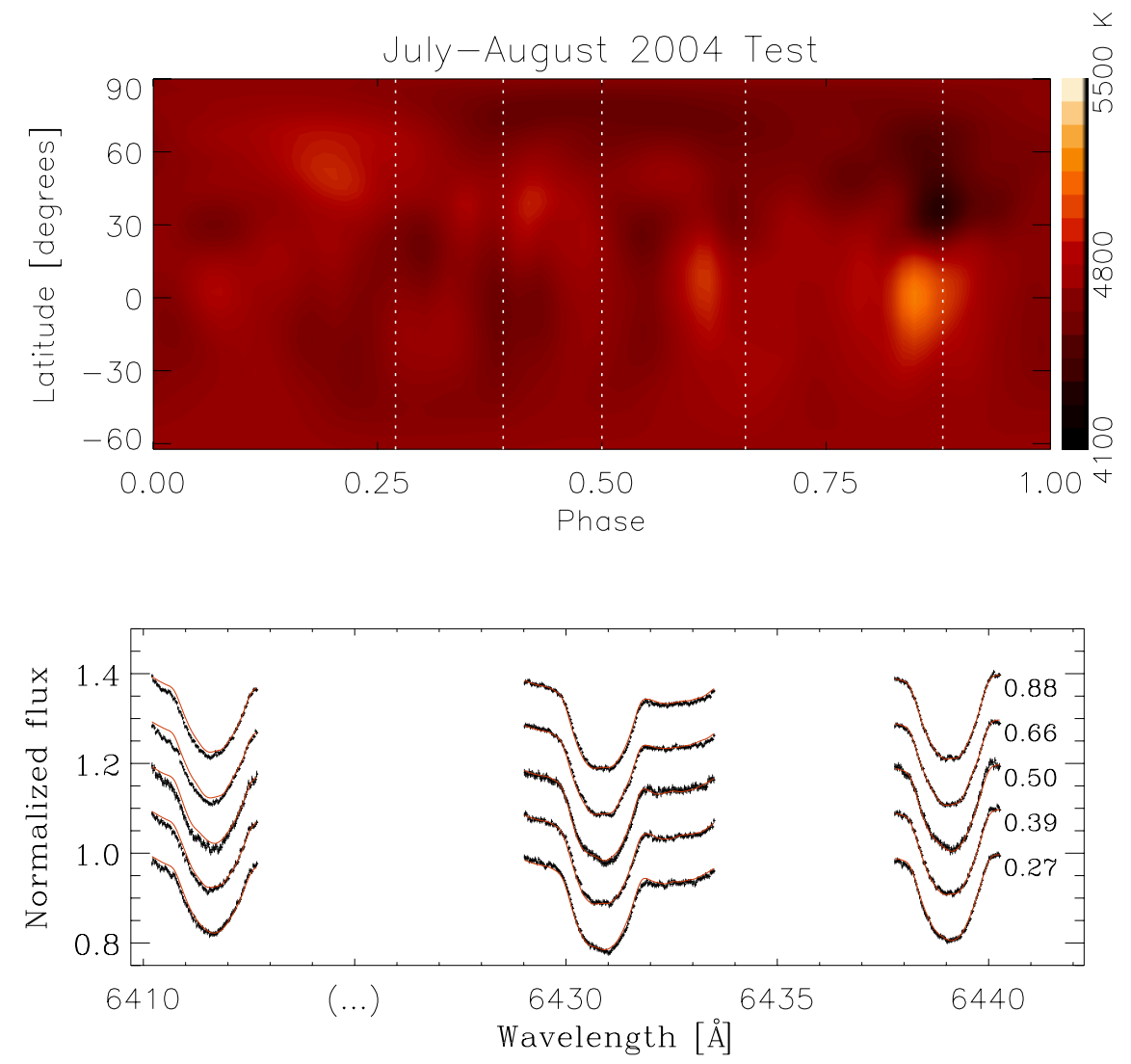

Fig. 3. Same as in Fig. 1 for a test using only five observed phases of the July-August 2004 data. 
Co $\equiv 4 \pi \mathrm{Ro}^{-1} \equiv 4 \pi \tau_{\mathrm{c}} P_{\text {rot }}^{-1} \approx 35$ for DI Psc. The estimated Coriolis number of roughly 35 places DI Psc at the high-Coriolis number edge on the "active" branch of the diagnostic diagram of Saar \& Brandenburg (1999), on which stars systematically show magnetic cycles of a few hundred times the rotational period, i.e. a few years to ten years. On this branch, the magnetic activity cycle length decreases as function of rotation rate. For comparison, in the Sun the Coriolis number is less than unity near the surface and of the order of 10 near the bottom of the convection zone (e.g. Käpylä et al. 2005). Even though this implies that the rotational influence on convection is far stronger in DI Psc than in the Sun, we are still not faced with the far extremes. For some other giant stars with similar spectral and luminosity classes, considerably higher Coriolis numbers can be deduced. For example, the RS CVn system's primary component, II Peg, for which $P_{\text {rot }} \approx 6.67$ (Berdyugina et al. 1999) and Co $\approx 94$, and the extremely rapid rotator FK Com (see e.g. Hackman et al. 2013, and references therein) with $P_{\text {rot }} \approx 2.4$ resulting in Co $\approx 261$. These extremely rapid rotators most likely fall, or at least approach, the "superactive branch" of the diagnostic diagram (magnetic activity cycle period as function of $\mathrm{Ro}^{-1}$; Brandenburg et al. 1998; Saar \& Brandenburg 1999) and are therefore expected to exhibit magnetic cycles that are several thousand times longer than the rotational period (several tens of years).

The expected relatively short magnetic cycle makes DI Psc an ideal target for Doppler imaging studies. Given that the star also shows a peculiar property related to its $\mathrm{Li}$-abundance, it is extremely fascinating to try to determine its approximate location in the above-mentioned diagnostic diagram.

The relatively large variations seen in the spot coverage fraction during only a few years can be at least considered to rule out the possibility of DI Psc exhibiting the very long magnetic cycle characteristic of the "superactive" branch or stars transiting towards it. The observational data gathered so far, however, is not adequate to distinguish whether the star belongs to the "active" or the "inactive" branch.

The connection between cool spots and magnetic fields in the Sun has seldom been detected in other stars, and it is not yet obvious that this relation can be extended to other types of cool active stars besides of the Sun (Kochukhov et al. 2013). A completely different scenario for the origin of the cool spots of rapidly rotating stars has been studied by Käpylä et al. (2011) and Mantere et al. (2011), who propose that the formation of temperature anomalies on the surfaces of rapidly rotating latetype stars could be generated by the hydrodynamical instability, creating large-scale vortices. This could also give another explanation for the hot spots near the large cool spots detected in several rapidly rotating stars, including DI Psc in this study, by Doppler imaging: these may not be artefacts, but actually a real phenomenon.

We observed DI Psc only in 2004-2006, so the time series is too short to detect activity cycles. The important future work would be to continue observing DI Psc in order to get longer time series of observations with better phase coverage to determine the magnetic cycle of the star.
Acknowledgements. This work has been supported by the Academy of Finland Centre of Excellence ReSoLVE (MM). The work of T.H. was financed through the project Active Suns by the University of Helsinki. H.K. acknowledges support by the European Commission under the Marie Curie IntraEuropean Fellowship Programme in FP7. O.K. is a Royal Swedish Academy of Sciences Research Fellow, supported by the grants from the Knut and Alice Wallenberg Foundation and Swedish Research Council. The authors are grateful to NORDITA for hospitality during their visits. The anonymous referee is acknowledged for valuable comments on the manuscript.

\section{References}

Balachandran, S. C., Fekel, F. C., Henry, G. W., \& Uitenbroek, H. 2000, ApJ, 542,978

Berdyugina, S. V. 2005, Liv. Rev. Sol. Phys., 2, 8

Berdyugina, S. V., Berdyugin, A. V., Ilyin, I., \& Tuominen, I. 1998, A\&A, 340, 437

Berdyugina, S. V., Ilyin, I., \& Tuominen, I. 1999, A\&A, 347, 932

Beuermann, K., Baraffe, I., \& Hauschildt, P. 1999, A\&A, 348, 524

Brandenburg, A., \& Subramanian, K. 2005, Phys. Rep., 417, 1

Brandenburg, A., Saar, S. H., \& Turpin, C. R. 1998, ApJ, 498, L51

Charbonnel, C., \& Balachandran, S. C. 2000, A\&A, 359, 563

Dall, T. H., Foellmi, C., Pritchard, J., et al. 2007, A\&A, 470, 1201

de La Reza, R., \& da Silva, L. 1995, ApJ, 439, 917

Drake, N. A., de la Reza, R., da Silva, L., \& Lambert, D. L. 2002, AJ, 123, 2703

Gray, D. F. 2008, The Observation and Analysis of Stellar Photospheres (Cambridge University Press)

Gustafsson, B., Edvardsson, B., Eriksson, K., et al. 2008, A\&A, 486, 951

Hackman, T., Mantere, M. J., Jetsu, L., et al. 2011, Astron. Nachr., 332, 859

Hackman, T., Mantere, M. J., Lindborg, M., et al. 2012, A\&A, 538, A126

Hackman, T., Pelt, J., Mantere, M. J., et al. 2013, A\&A, 553, A40

Ilyin, I. V. 2000, Ph.D. Thesis, Astronomy Division Department of Physical Sciences, University of Oulu, Finland

Jeffers, S. V., Donati, J.-F., Alecian, E., \& Marsden, S. C. 2011, MNRAS, 411, 1301

Käpylä, P. J., Korpi, M. J., Stix, M., \& Tuominen, I. 2005, A\&A, 438, 403

Käpylä, P. J., Mantere, M. J., \& Hackman, T. 2011, ApJ, 742, 34

Kővári, Z., Korhonen, H., Strassmeier, K. G., et al. 2013, A\&A, 551, A2

Kochukhov, O., Mantere, M. J., Hackman, T., \& Ilyin, I. 2013, A\&A, 550, A84

Koen, C., \& Eyer, L. 2002, MNRAS, 331, 45

Korhonen, H., Berdyugina, S. V., Hackman, T., et al. 2007, A\&A, 476, 881

Kumar, Y. B., Reddy, B. E., \& Lambert, D. L. 2011, ApJ, 730, L12

Kupka, F., Piskunov, N., Ryabchikova, T. A., Stempels, H. C., \& Weiss, W. W. 1999, A\&AS, 138, 119

Lindborg, M., Korpi, M. J., Hackman, T., et al. 2011, A\&A, 526, A44

Lindborg, M., Mantere, M. J., Olspert, N., et al. 2013, A\&A, 559, A97

Mantere, M. J., Käpylä, P. J., \& Hackman, T. 2011, Astron. Nachr., 332, 876

Mantere, M. J., Käpylä, P. J., \& Pelt, J. 2013, Proc. IAU Symp., 294, 195

Melo, C. H. F., de Laverny, P., Santos, N. C., et al. 2005, A\&A, 439, 227

Ossendrijver, M. 2003, A\&AR, 11, 287

Pelt, J., Brooke, J. M., Korpi, M. J., \& Tuominen, I. 2006, A\&A, 460, 875

Piskunov, N. E. 1991, in IAU Colloq., 130, The Sun and Cool Stars. Activity, Magnetism, Dynamos, eds. I. Tuominen, D. Moss, \& G. Rüdiger, Lect. Not. Phys. (Berlin: Springer Verlag) 380, 309

Piskunov, N. E., Tuominen, I., \& Vilhu, O. 1990, A\&A, 230, 363

Reddy, B. E., \& Lambert, D. L. 2005, AJ, 129, 2831

Rice, J. B., \& Strassmeier, K. G. 2000, A\&AS, 147, 151

Saar, S. H., \& Brandenburg, A. 1999, ApJ, 524, 295

Strassmeier, K. G. 2009, A\&AR, 17, 251

Strassmeier, K., Washuettl, A., Granzer, T., Scheck, M., \& Weber, M. 2000, A\&AS, 142, 275

van Leeuwen, F. 2007, A\&A, 474, 653

Vogt, S. S., Penrod, G. D., \& Hatzes, A. P. 1987, ApJ, 321, 496

Washuettl, A., Strassmeier, K. G., \& Weber, M. 2009, Astron. Nachr., 330, 366 